\title{
Structure-function characterisation of Chlamydia pneumoniae MOMP
}

Amy Elizabeth Danson ${ }^{1}$, Isabel de Moraes ${ }^{2}$, Martin Walsh ${ }^{2}$, Sheila MacIntyre ${ }^{1}$, Kimberly Watson ${ }^{1}$

${ }^{1}$ School Of Biological Sciences, University Of Reading, Reading, United Kingdom, ${ }^{2}$ Diamond Light Source, Oxfordshire, United Kingdom

E-mail: a.e.danson@pgr.reading.ac.uk

Major outer membrane proteins (MOMPs) are structurally and immunologically dominant proteins in the Chlamydia outer membrane. Both the surface exposure and abundance of MOMP suggests a possible role in Chlamydia-host interactions, highlighting MOMP as a possible vaccine target. The proposed beta-barrel structure, derived from homology modelling with FadL of E. coli [1], also indicates a role in ligand transport. Due to the large number of cysteine residues within MOMP and the lack of detectable peptidoglycan in Chlamydia, it is also hypothesised that MOMP may have a structural role in forming disulphide bridges with other cysteine rich proteins, creating a stable compensatory mesh. Currently, there is very little literature surrounding structure-function characterisation of Chlamydial MOMPs due to difficulties in producing recombinant protein in a folded, active state. Therefore, this research is focussed on using X-ray crystallography to solve MOMP's structure, in cohort with ligand assays to assess functionality, and mutagenesis studies to investigate the role of the cysteine residues.

Synchrotron radiation circular dichroism (SRCD) and Fourier transform infrared radiation spectroscopy data have been used to provide a more detailed insight into MOMP's structure-function properties. Both techniques revealed that MOMP is predominantly a beta-sheet protein, with SRCD demonstrating that MOMP's thermal stability is increased in the presence of fatty acids (FAs). X-ray crystallography has revealed a low-resolution crystal structure of $4 \AA$, from non-optimised crystals, that shows a beta-barrel structure with an occluded pore. Preliminary course grained molecular dynamics simulations have shown that two MOMPs modelled in an asymmetrical lipid bilayer can form stable clusters. Improved resolution of the crystal structure will permit the use of more detailed atomistic modelling, which will extend the computational analysis of MOMP by simulating ligand transport. To complement this, functional assays are in progress to assess MOMP's potential activity as a FA transporter, due to observed thermal stabilisation in the presence of FAs as well as its structural resemblance to the FA transporter FadL of $E$. coli. The structural role of the cysteine residues within MOMP will be investigated through mutagenesis studies.

Currently, little is known regarding the details of the structure and function of MOMP, the most abundant protein in the Chlamydia membrane. Research into the functional and structural characteristics of this outer membrane protein will provide novel insight into its role, potentially laying down critical foundations for future immunological studies. Due to their conserved nature, this research is also applicable to other Chlamydial MOMPs, in addition to other cysteine rich proteins.

[1] Atanu, F.O. et al. (2013) PloS one. 8(4), e61139

Keywords: major outer membrane protein, MOMP, Chlamydia pneumoniae 